\title{
Optimization of a Fabry-Perot Sensing Interferometer Design for an Optical Fiber Sensor of Hematocrit Level
}

\author{
P. WiERzBA AND M. JĘDRZEJEWSKA-SzCZERSKA* \\ Department of Metrology and Optoelectronics, Faculty of Electronics, Telecommunications and Informatics \\ Gdańsk University of Technology, G. Narutowicza 11/12, 80-233 Gdańsk, Poland

\begin{abstract}
Continuous measurement of the hematocrit level in blood can potentially be performed using optical fibre sensors. The Fabry-Perot interferometric sensors are a promising candidate in this application. The most important step in the design of the sensor is design of the sensing interferometer. Adequate cavity length and high interference contrast are two most important requirements in this application. The design method of the sensing interferometer presented in this paper uses a Gaussian beam approximation. In order to verify the design, an optical fibre FabryPerot interferometer with adjustable cavity length was built. Its performance was tested, confirming validity of the design approach.
\end{abstract}

DOI: $10.12693 /$ APhysPolA.124.586

PACS: 07.60.Ly, 03.75.Dg, 42.81.Pa, 07.60.Vg

\section{Introduction}

The hematocrit (HCT) level in human blood provides important information about the patient's health. Continuous monitoring of this parameter is desirable in several cases of medical therapy and diagnosis. Currently, however, it is difficult to conduct, as the standard procedure require blood to be sampled and processed in laboratory. Optical fibre sensors are potentially capable of continuous monitoring of the HCT level. Until now most of research in this area have been focused on spectral measurement techniques [1]. However, optical-fiber FabryPerot interferometric sensors offer potentially more robust solution, as the information about measurand is encoded in full spectrum, rather than in few wavelengths.

One of the most important steps of the design process of such a sensor is design of the sensing Fabry-Perot interferometer. Therefore, the objective of this paper is to present the requirements and method employed in the design of a Fabry-Perot interferometer for an optical fiber sensor of the HCT level. Section 2 gives an outline of theory describing the operation of such interferometer and presents the proposed design method. Experimental verification of the is described in Sect. 3. Finally, conclusions are formulated in Sect. 4.

\section{Optical-fiber Fabry-Perot interferometer}

A Fabry-Perot interferometer built from bulk optical components is shown in Fig. 1a ([2], p. 329). It consists of two flat transparent plates $\mathrm{P}_{1}$ and $\mathrm{P}_{2}$, parallel to each other, separated by distance $d$ with reflective layers $\mathrm{L}_{1}$ and $\mathrm{L}_{2}$ deposited on their inner surfaces. Derivation of expressions for intensity of light reflected from and transmitted by this interferometer is usually performed under three assumptions: $\left(1^{\circ}\right)$ plane wave illumination

*corresponding author; e-mail: mjedrzej@eti.pg.gda.pl of the interferometer, $\left(2^{\circ}\right)$ equal reflectivity of layers $\mathrm{L}_{1}$ and $\mathrm{L}_{2},\left(3^{\circ}\right)$ no loss in the cavity. Such derivation yields well-known classic formulae $[3,4]$.

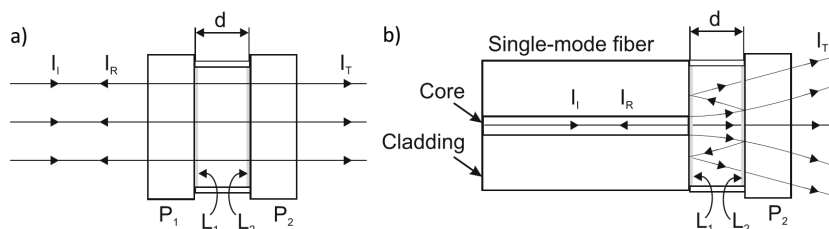

Fig. 1. Fabry-Perot interferometer. (a) Interferometer made from bulk optical components, (b) Fabry-Perot interferometer illuminated by single-mode fiber, where $I_{\mathrm{I}}$ - intensity of incident beam, $I_{\mathrm{R}}$ - intensity of reflected beam, $I_{\mathrm{T}}$ - intensity of transmitted beam, $\mathrm{P}_{1}$, $\mathrm{P}_{2}$ - plane-parallel glass plates, $\mathrm{L}_{1}, \mathrm{~L}_{2}$ - reflective layers.

In contrast, the optical fiber Fabry-Perot interferometer, shown schematically in Fig. 1b, is illuminated by a divergent beam from a single-mode fiber. Its reflective layers $\mathrm{L}_{1}$ and $\mathrm{L}_{2}$ may have different reflectivity. Consequently, the classic formulae do not hold.

Let us assume that layers $\mathrm{L}_{1}$ and $\mathrm{L}_{2}$ as well as the medium in the interferometer cavity are non-absorbing. Reflection coefficients of these layers, defined as the ratios of reflected to incident amplitudes of electric field vectors, are $r_{1}$ and $r_{2}$, respectively.

Light exiting the fibre can be described using Gaussian beam formalism [5]. The waist of the beam is located on the end surface of the fibre, i.e. in reflective layer $L_{1}$. The diameter $2 W_{0}$ of the beam in the waist is equal to the mode-field diameter (MFD) of the fibre.

Coupling loss coefficient $\alpha(x, n)$ can be defined as:

$$
\alpha(x, n)=\frac{A_{\mathrm{R}}(x, n)}{A_{\mathrm{I}}},
$$

where $A_{\mathrm{I}}$ - amplitude of the beam incident on the inter- 
ferometer, $A_{\mathrm{R}}$ - amplitude of the beam coupled back to the fiber, $n$ - refractive index of the medium filling the interferometer cavity, $x$ - distance propagated by the beam in the interferometer. It can be assumed that the coupling loss coefficient $\alpha$ decreases with $x$ at the same rate as the amplitude of the Gaussian beam propagating in the interferometer, i.e.

$$
\alpha(x, n) \sim\left[1+\left(\frac{x}{x_{0}}\right)^{2}\right]^{-\frac{1}{2}} .
$$

As a result of multiple reflections in the cavity, a series of beams are coupled back to the fiber. Their amplitudes can be expressed as

$$
\begin{aligned}
& A_{1}=r_{1} A_{\mathrm{I}} \\
& \quad \vdots \\
& A_{M}=r_{2}^{M-1} r_{1}^{M-2}\left(1-r_{1}\right)^{2} \alpha\left(2(M-1) x_{\mathrm{FP}}, n\right) A_{\mathrm{I}} \\
& \quad \text { for } M \geq 2,
\end{aligned}
$$

where $A_{\mathrm{I}}$ - the amplitude of the incident beam; $A_{i}-$ the amplitude of $i$-th reflected beam, $r_{1}, r_{2}$ - reflection coefficients of $\mathrm{L}_{1}$ and $\mathrm{L}_{2}$, respectively, $\alpha-$ coupling loss coefficient, $n-$ refractive index of the medium in the cavity, $x_{\mathrm{FP}}$ - length of the Fabry-Perot cavity. Phase difference $\delta$ between $i$-th and $i+1$-th beam is

$$
\delta=4 \pi \frac{n x_{\mathrm{FP}}}{\lambda} .
$$

$n$ - refractive index of the medium in the cavity, $x_{\mathrm{FP}}-$ length of the cavity, $\lambda$ - wavelength.

The complex amplitude $A_{\mathrm{R}}$ of the sum of reflected beams is given by

$$
A_{\mathrm{R}}=A_{1}+A_{2} \mathrm{e}^{-\mathrm{i} \delta}+\ldots+A_{N} \mathrm{e}^{-\mathrm{i} N \delta},
$$

where: $\delta$ - phase difference given by (4), $A_{N}$ - the amplitude of $N$-th reflected beam.

Because of the presence of coupling loss coefficient $\alpha$ in $A_{i}$, the amplitudes $A_{i}$ decrease faster than those of the same Fabry-Perot interferometer illuminated by a plane wave. Consequently, the number of beams effectively contributing to the interference is smaller that that in the plane wave-illuminated interferometer case.

A Fabry-Perot interferometer designed for investigation of the hematocrit (HCT) level should operate in the reflection mode to simplify the setup. It should have the cavity length $x_{\mathrm{FP}}$ much bigger than the maximum dimension of any blood cell. Good interference contrast $V$ of the reflected signal is advantageous in detection of the signal and its subsequent processing. High reflectivity $R$ of the interferometer's layers is not required, and can be as low as $1 \%$, while retaining adequate signal-to-noise ratio at the output of the detection setup.

Design of the interferometer required calculating optimum values of the cavity length $x_{\mathrm{FP}}$ and reflection coefficients $r_{1}$ and $r_{2}$. To simplify the design, only two reflected beams $A_{1}$ and $A_{2}$ were considered. Neglecting the remaining beams reduces $V$, but the reduction was expected to be acceptably low.

The highest interference contrast, $V=1$, is attained only when $A_{1}=A_{2}$. Using (2) this condition can be ex- pressed as

$$
r_{1}=r_{2}\left(1-r_{1}\right)^{2} \alpha\left(2 x_{\mathrm{FP}}, n\right),
$$

where $r_{1}, r_{2}$ - reflection coefficients, $\alpha$ - coupling loss coefficient, $n$ - refractive index of the medium in the cavity, $x_{\mathrm{FP}}$ - length of the Fabry-Perot cavity. Equation (6) can be written in the form better suited for the design process, i.e. as

$$
\alpha\left(2 x_{\mathrm{FP}}, n\right)=\frac{r_{1}}{r_{2}\left(1-r_{1}\right)^{2}} .
$$

From Eq. (2) follows that increasing the length of the Fabry-Perot cavity $x_{\mathrm{FP}}$ corresponds to decreasing $\alpha$. Accomplishing this without reducing $V$ requires, as it can be seen from (7), increasing $r_{2}$ and decreasing $r_{1}$. While $r_{2}$ can be increased close to 1.0 , the lower limit on $r_{1}$ is set by minimum required reflectivity $R_{\mathrm{MIN}}$ :

$$
R_{\mathrm{MIN}}=4 r_{1}^{2} \text {. }
$$

For $R_{\mathrm{MIN}}=0.05, r_{1}$ is 0.036 . Therefore, the Fresnel reflection at the boundary between the fiber and the medium in the Fabry-Perot cavity can be used, if the refractive index of this medium is below 1.35.

\section{Experimental}

The measurement system employing the designed Fabry-Perot interferometer is shown in Fig. 2. It consists of a superluminescent diode (Superlum Broadlighter S1300-G-I-20, Gaussian spectral density, $\lambda_{\mathrm{MAX}}=$ $1290 \mathrm{~nm}, \Delta \lambda_{\mathrm{FWHM}}=50 \mathrm{~nm}$ ) acting as a light source, connected to the Fabry-Perot interferometer by a single-mode SMF-28 fiber, and an Ando AQ6319 optical spectrum analyzer with resolution bandwidth set to $1 \mathrm{~nm}$ working as the detection setup [6]. Acquired spectra are processed by a computer connected to the spectrum analyzer.

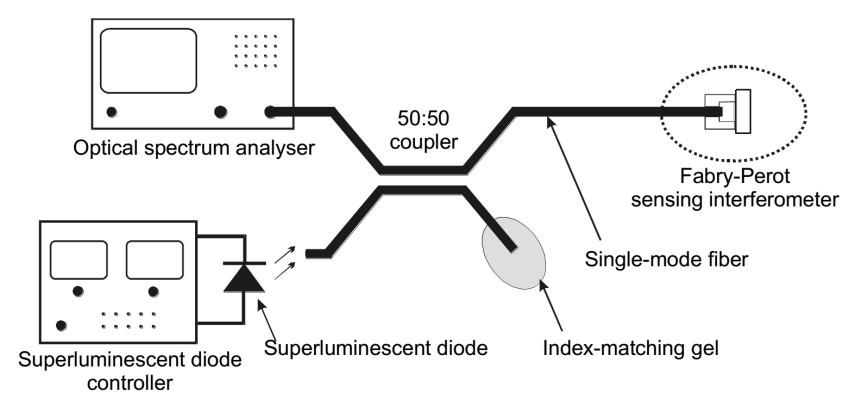

Fig. 2. The measurement system.

A fibre-optic Fabry-Perot interferometer was built, as shown in Fig. 3. The cavity was formed by the uncoated end surface of the single-mode fiber $\left(r_{1}=0.2\right)$ and the silver mirror $\left(r_{2}=0.995\right)$. The fiber, attached to a translation stage equipped with a differential adjuster, moved in the silica glass capillary, changing the cavity length $x_{\mathrm{FP}}$.

The response of the interferometer (i.e. the spectrum acquired by the optical spectrum analyser) was recorded for several values of cavity length $x_{\mathrm{FP}}$. Example results are shown in Fig. 3. 


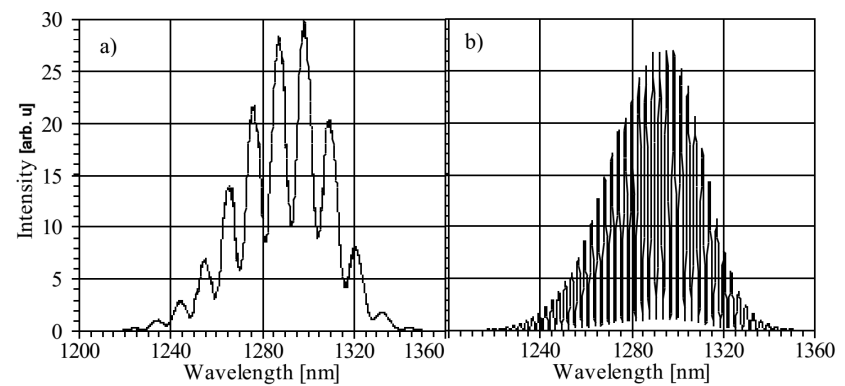

Fig. 3. Spectrum reflected from the interferometer for cavity length: (a) $x_{\mathrm{FP}}=150 \mu \mathrm{m}$, (b) $250 \mu \mathrm{m}$.

Following, visibility was calculated for each measured spectrum and is shown in Fig. 4, along with visibility predicted using the theory from the previous section.

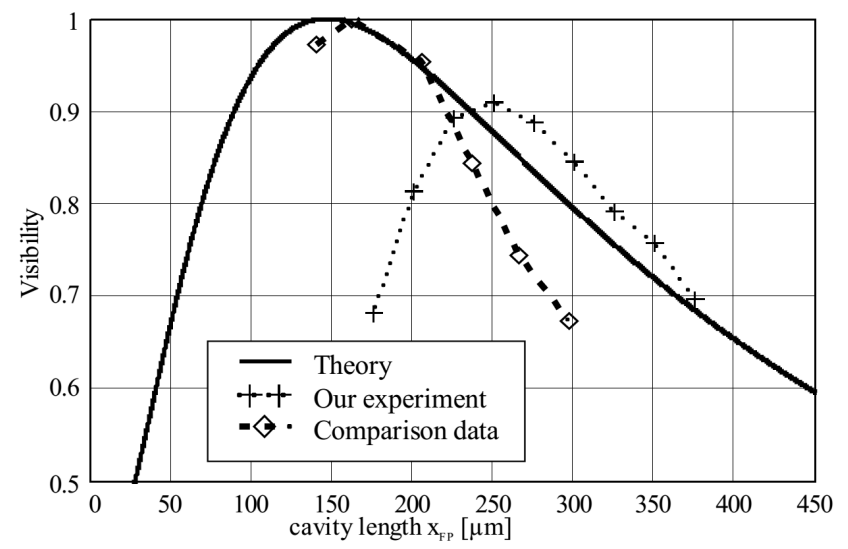

Fig. 4. Visibility as a function of the cavity length $x_{\mathrm{FP}}$.

Comparing the two sets of data, one can note that the experimental and theoretical data agree reasonably well when the cavity length $x_{\mathrm{FP}}$ is greater than $225 \mu \mathrm{m}$. When the cavity is shorter, visibility drops rapidly. This can be caused either by (a) more than two beams effectively interfering in the cavity or by (b) behaviour of the coupling loss coefficient $\alpha$ different from that assumed in the previous section. In order to identify the source of the discrepancy, experimental data from [7] was used. The interferometer studied in [7] was also illuminated with a single-mode fiber and had two reflective surfaces using glass-air boundary. By assuming that only two beams effectively interfered in its cavity, the measured visibility given in Fig. 5 of [7] was recalculated for the interferometer designed in the previous section and it is shown in Fig. 4 as the comparison data. This data shows good agreement with the theoretical data. The agreement could be even better if some adjustments of theoretical model parameters were made within the tolerances for SMF-28 fiber parameters. Therefore, if only two beams interfere in the cavity, the result of that interference follows our theoretical prediction. This means that the behaviour of the coupling loss coefficient $\alpha$ is consistent with that assumed in the previous section, and the reduction in the visibility observed in our experimental data is caused by more than two beams effectively interfering in the cavity.

\section{Conclusions}

A design method for a low-finesse Fabry-Perot sensing interferometer illuminated by a single-mode fibre is described. A Fabry-Perot interferometer was designed using this method and its performance was evaluated. Obtained measurement data differ markedly from the results of design calculations. This difference is attributed to the fact that the designed interferometer cannot be accurately described using two-beam model. However, presented design method provides good agreement with experimental data when the cavity length $x_{\mathrm{FP}}$ is greater than $225 \mu \mathrm{m}$. Designed interferometer will be subsequently employed in an optical fibre sensor for assessment of hematocrit level in blood.

\section{Acknowledgments}

This study was partially supported by the National Science Center under the grant no. 2011/03/D/ST7/ 03540, titled: "Investigation of the relationship between the spectrum of optical signal and blood properties" as well as DS Programs of the Faculty of Electronics, Telecommunications and Informatics, Gdańsk University of Technology.

\section{References}

[1] J.M. Schmitt, G.-X. Zhou, J. Miller, Proc. SPIE 1641, 150 (1992).

[2] M. Born, E. Wolf, Principles of Optics, 6th ed., Pergamon Press, Oxford 1993.

[3] P. Hariharan, Optical Interferometry, AcademicElsevier Sci. San Diego 2003.

[4] M. Jędrzejewska-Szczerska, M. Gnyba, B. Kosmowski, Acta Phys. Pol. A 120, 621 (2011).

[5] D. Marcuse, Light Transmission Optics, 2nd ed., Van Nostrand Reinhold Co., New York 1982, p. 339.

[6] T. Liu, D. Brooks, A. Martin, R. Badcocko, G. Fernando, Proc. SPIE 2718, 408 (1996).

[7] V. Arya, M. de Vries, K.A. Murphy, A. Wang, R.O. Claus, Opt. Fib. Tech. 1, 380 (1995). 